\title{
ANALYSIS OF UNCERTAINTY IN A CONSTRUCTION PROJECT
}

\author{
Dr. A. Paul Makesh \\ Associate Professor, Department of Civil Engineering, Wolaita Sodo Univeristy, Sodo, \\ Ethiopia \\ E-mail: paulmakesh1986@gmail.com
}

\begin{abstract}
This article presents the analysis of uncertainty and effectiveness in cost to construction project. Theoretical part describes various definitions of uncertainty, risk both with there sources as calculation bias, information ambiguity and data inaccuracy in construction projects. Main analysis is connected with the project's appraisal phase and its stages were the detailed information is the crucial factor for correct decision- making procedures under uncertainty and risk. Consequently this article is presenting various types risk what are undistinguishable from uncertainty circumstances in construction. After the description of risk and uncertainty types list of solutions were prepared. Application of the suggested risk and uncertainty extenuating techniques in practice was shown as analysis of precision and bias dependence with available information at different stages of appraisal phase.It is emphasized on uncertainty and risk management problem in the construction's decision-making. Recently methods based on the utility theory, game theory, statistical distribution and probability, were improved and adjusted due to decision-making needs in construction. That is why the model with implemented multiple-criteria approaches is suggested both as a tool for dealing with the uncertainty and risk problems and to meet the needs of project managers in appraisal phase.
\end{abstract}

Keywords: Risk Management, Uncertainty, Planning, Scheduling.

\section{Introduction.}

One of the concepts that more effectiveness has demonstrated in the management of construction projects is the concept of Lean. The idea of Lean is to eliminate what does not add value from the perspective of the customer. In this analysis, the implications and consequences that the use of certain risk management methods have in Lean Construction are assessed.

In addition, it is essential to improve the projects management by identifying the risks, planning responses and coordinating the information flow and the resources. In order to give the widest range of solutions to this, three important methods are evaluated: the TFV-model (transformation, flow and value), the Last Planner System and the Location-Based Scheduling; concepts that are connected and used to mitigate the possible damage inflicted by unexpected 
situations.In this approach, the correlation and development of these methods in the construction industry are also analyzed. From early phases, the overall project should be defined: scheduling, planning, cost, time and resources. But there is other information, a level of uncertainty, which needs to be recorded. Uncertainty can be defined as the difference between the information required to make a decision and the information available. There are two concepts attached to this.

- Predictability, since the future is not known, and the past can be an invaluable guide.

- Complexity, since it is costly in terms of time, money and other resources.

Risk is the probability of the occurrence of a risk event given its risk source. To define risk, two definitions should be considered:

- Risk source, as an underlying state of affairs

- Risk event, as something given due to the underlying state of affairs

When the needs of the owner and occupants of a building are not met, a risk event can be created from the design phase. In addition, modifying these needs during the project, or having a weak communication between the parts, can create the risk event. In risk situations, there are parameters controlled by probability, known by the decision maker ${ }^{[2]}$. However, in uncertainty situations, parameters are uncertain and no information about probabilities is known. It is clear that construction management organizations do not have precise information, especially when making long-term planning. But it is possible to determine some parameters from experience or statistical data. The uniqueness of a construction project leads to a high degree of uncertainty. Therefore, project managers must lean on risk management to identify, analyze, monitor and report risks. The project risk management uses four basic concepts to help identify the type of risk:

\section{Risk and uncertainty in construction industry}

The term uncertainty is used in most of scientific literature concerning risk management. Theoretically it can be defined as a lack of certainty involving variability and/or ambiguity. Alike uncertainty management is concerned as managing perceived threats and opportunities and their risk implications but also managing the various sources of uncertainty which give rise to and shape risk, threat and opportunity Chapman and Ward [4]. In risk situations, there are uncertain parameters controlled by probability distributions are known by the decision maker [2]. Whereas in uncertainty situations, parameters are uncertain, and furthermore, no information about probabilities is known. Problems in risk situations (stochastic optimization problems) intent to optimize the expected value of some objective function and problems under uncertainty (robust optimization problems) often attempt to optimize the worst-case performance of the system. However, goal of both stochastic and robust optimization is to find a solution that will perform well under any possible realization of the random parameters. In our case both describes uncertainties in construction project management. It is clear that construction management organizations do not have exhaustive and precise information, especially regarding making long-term plans. It is possible to determine some parameter probability characteristics from experience or statistical data in certain situations of process's 
research. But in other situations there is no data on which it would be possible to determine statistical peculiarities of parameters in risk-related and uncertain situations. In practice probability parameters are considered to be known and equal to average of probable result. However, this substitution of probability processes with determined models is not always right. Stochastic programming is planning and management tasks which describe processes going under risk and uncertainty conditions, it examines tasks with random coefficients and finds solutions when the information on the task conditions is insufficient. In compilation of stochastic problems and their analysis it is very important to know if it is necessary to get only one solution which cannot be changed or a solution which can be adjusted [7] according to newly obtained information (two-step and multi-step task).

Every construction project is unique, with unique data, environment and includes a high degree of information's uncertainty. All construction projects experience the unexpected situations and uncertainties, due their implementation, what is why project managers must be ready for it. They must be supported by risk management to identify, analyze, control, and report risk. [8] The project risk management explains this uncertainty phenomenon [9-10] using three basic sources [2, 4]: Known-unknowns are explicit assumptions or conditions which, if not valid, could have uncertain, significant consequences and can be analyzed and managed. Represent identified potential problems, such as possibility of a strike when a labour contract expires or enough rain to stall a construction project during winter. We don't know exactly what will happen, but we do know it has a potential to damage our project and we can prepare for it.

\section{Transformation, Flow and Value}

Lean Construction consists of several tools, which enable all parties to perform their tasks without compromising the resources. The TFV-model illustrates the different outlooks in project management. In order to discuss the parts of the TFV-model, it is necessary to differentiate the effectiveness, which is to do the right thing, and efficiency, which is to do it right.

This model consists of three concepts:

- Transformation: when the workers are told to do something, but not how to do it. This focuses on effectiveness, leading to low efficiency.

- Flow: the waste between processes is eliminated. It is important to know what to do, before knowing how to do it properly.

- Value: there is a focus on creating value for the client. Here is when the project management should be used. 


\subsection{Last Planner System}

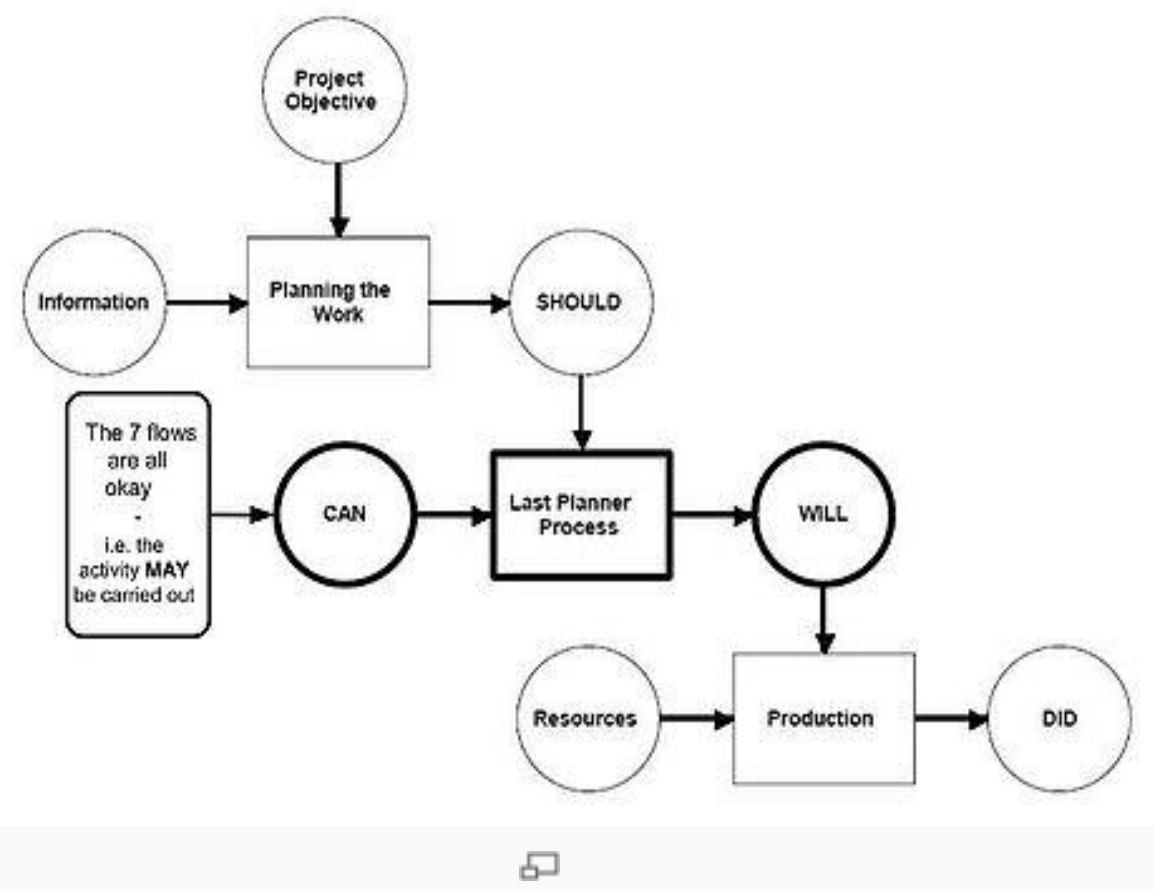

Figure 1 : Illustration of the Last Planner System

In order to facilitate the TFV-model, the LPS was developed for construction purposes. When managing a construction project traditionally there is a tendency to start the work, even if not all the resources required for the completion of the task are available. The Last Planner System addresses this problem with a break down structure of the schedule. The method consists in dividing the construction process into three levels: phase, look-ahead and weekly work plan. This is done to provide an even workflow which enables the prediction of durability of the activities.

Firstly, a phase schedule is created, containing the overall construction project with the corresponding deadlines. Then, each representative is required to create a look-ahead schedule to clarify how the activities in the phase schedule should be done. Finally, weekly work plans are created during weekly meetings with the representatives. In these meetings, agreements are done about what to do on that week. The LPS produces predictable workflow and rapid learning ${ }^{[5]}$. This creates the maximum value for the owner by eliminating waste caused by unpredictable workflow. Moreover, it enables contractors to reduce the delivery time of the project and creates commitments among project participants.

But even with the situation in which the schedule makes the weekly work plans efficient, there is another aspect to consider when the foremen are doing the planning on site: the weekly meetings are also oriented to commitment and trust among the parties ${ }^{[6]}$. This is achieved by letting the workers do the planning and adjust their own management to the overall project ${ }^{[7]}$. This way, an open and easy way to communicate is created. In the Figure 1, the information flow and LPS process are shown. Regarding the concept of the 7 flows, which is part of this 
figure, it is used to represent the conditions that must be analyzed and met in order to perform an activity in a construction project:

- Prerequisite work: must be done

- Materials: must be ready

- Equipment: must be ready

- Personnel: must be qualified

- Right information: must be at hand

- Space: must be appropriate and safe

- External circumstances: should be clarified

\subsection{Location-Based Scheduling}

In response to the limitations of the Last Planner System, the Location-Based Scheduling method was created. This enables a more detailed schedule to be established in the design phase, where activities can be performed at the same time, reducing waste of time and resources.

This method aims to secure that under or overloading of resources does not occur. Research studies affirm that LBS is especially advantageous in terms of:

- Understanding the overall work plan and communication with sub-contractors and suppliers.

- Efficient resource flow.

- Evaluation and quality assurance of the executed work.

This follows the line of Lean philosophy and shares the advantages of the Last Planner System. It seems logical to assume that the efficient flow of resources in LBS will decrease the level of uncertainty, and therefore, decrease the variability of task durations. This being said, it can be stated that the LBS seems to be an adjunct to the LPS, rather than a replacement.

Locations are important in the construction industry since it is not a systematic repetitive process, but a series of physical locations in which tasks are variable and of different type and quatity ${ }^{[8]}$. The location is the container for data which relates to the project. The LBS is hierarchical so that a higher level location includes all the data of the lower level locations. Each of the locations have a different purpose and the highest level is used to optimize the construction sequence. Since the structures of such sections are independent of each other, it is possible to start them simultaneously. The middle levels are used to plan the production flows, and the lowest levels for planning the finishes. This allows the data to be collected at different levels. The location consists of the following data:

- Building objects: components such as elements and sub-systems, that should be included, not only in drawings, but also as a 3D object oriented construction model.

- Planned building component quantities: quantities measured from a 3D model. This way, variations in quantity which occur during construction should be able to be tracked.

- Building system assemblies: this includes the construction method, support components and building site planning.

- Material costs: cost related to the planned material quantities.

- Building system costs: costs of the components that must be included in each location. The labour costs should be calculated based on the actual resource available in the plan. 
While designing according to the data of a technical project the following questions are being solved:

- Local decisions for designing are being made (identification of missing information and supplementing the project with local decisions);

- A variance/variability designing is performed: it is being searched for a rational set of building's constructional versions with alternative analysis.

Risk management operate throughout the project life cycle but a disproportionate part occurs during the appraisal phase. During appraisal, a relatively small amount of money is invested to fund the work of the project management team. However the solutions choose and decisions made in this stage have crucial influence to whole implementation of the project and need to be done by qualified person with high level of expertise and responsibility.

There is no standard method for making this decision, a wide range of tools and special techniques [19] are available to assist the project manager by providing better information. Ustinovicius [16],[20] stated what this is serious problem to decision support approaches, because it is not clear which method fits a particular construction problem better [21]. Scientists in their research noticed what it is important to choose right normalization method and to follow up with this point it must be done according to the problem objectives, special requirements and with regard to possible inaccuracy or uncertainty threats [6]. At the end best computer techniques and the comprehensive analysis will be just support to the final decision has to be made by the project manager within the overall strategy of the company and goals of the project

\section{Need of reducing the risk and uncertainty}

A traditional construction project can be described as a model covering all stages of its implementation [22], [23]: development and planning, design and economic assessment, tender and negotiations, construction and handover, maintenance and utilization. In these stages certain participants (Customer, costumer's representative, department, designer, contractor, investor or owner) perform the appropriate actions. The information collected through earlier stages [24] is transferred to the next stages.

The lack of information exchange among the project participants negatively affects the implementation of the construction project [2]: increasing the execution times, being the reason for the demand of non-scheduled resources, including ruined human resource management (HRM) and planned resource supply chain. It is possible to imagine technical, economical or a daily life situation where the consequences of not fulfilling of one or another restriction can be very unwelcoming or even tragic. In the model of such tasks it should be required that a set of variable parameters would satisfy all project's limitations in a presence of any kind of fortuitous coefficients.

Kleim and Ludin [8] spotlighted several factors what can raise risk: team size; history and project similarity; staff expertise and experience; complexity; management stability; time compression; resource availability. Uncertainties often rise due to the lack of qualification and competence of the personnel from the project manager's team. Qualification and competence of 
EU workers has been reviewed and the importance of appraisal and planning processes has been emphasised in The Leonardo Da Vinci project [1] "Recognition of needs and creation of the professional training in the area of the preparation and management of infrastructure construction projects financed by the European Union". So the uncertainty in undertaking a construction project comes from many sources and often involves many participants in the project [17]. Since each participant tries to minimize its own risk, the conflicts among various participants can be crucial to the project. Failure to recognize this responsibility by the client or project manager often leads to undesirable results. Uncertainties related with Force Major situations cannot be evaluated in project evaluation, because the factors of an influence can be disastrous and the project won't be available to implement.

Estimation and evaluation of uncertainty are core tasks in any decision support process [19]. The greatest influence to construction cost estimation (appraisal procedures) occurs at the front end of the project (illustrated in Figure 1) [2].

\section{Methodology, Analysis and Implementation}

\subsection{Improper understanding of scope:}

We may not have considered all the requirements. For example, We may not consider few basic things like, Do we need to replace the baseboards? If we don't consider the replacement of baseboards, our plan is missing an important component, and our estimation will not include the work of replacing baseboards.

\subsection{Incomplete understanding of effort for work:}

Sometimes we consider the work properly, but we will have to consider them at the top level only and miss out on small processes/things included in the actual process. For example, consider that we included baseboard replacement in our plan or scope, but we consider that the effort involved was limited to replace the new ones in the position of old ones.Unfortunately, we didn't count for the work required to measure and cut the baseboards to the right size and right shape. So, here even we estimated the scope correctly, but we failed to estimate the effort required and considered it as too low.

\subsection{Improper Understanding of Known work:}

Okay, for instance, consider that we estimated the effort also. Even though we do remember all of the work that needs to be accomplished for the work of replacing the baseboards, and estimate correctly. Our estimates may be wrong because some of the boards may damage while replacing. And we will either have to replace those baseboards again or need to drill them to avoid splitting when we attach them. In both ways, the work will increase our estimation. Unable to predict the unexpected: Under this case, we have to question ourselves with "what if?" or "what happens if?" What happens if the material we need is out of stock, or what if someone delivers the wrong baseboards? So these types of external events are uncertain and may deviate from our schedule. 


\section{Evaluation of alternatives and analysis results}

In this paper, the research is focused on the integration of multiple criteria decisions into computer- aided modelling-management-evaluation systems as well as using the algorithm of the synthesis methods [29], for combining several construction projects or a few phases of design stages into a joint system [24]. The researchers Peldschus, Zavadskas, Ustinovichius developed decision support software LEVI 3.0 [5]. Using this software, it is possible to find solutions to problems by different methods, to compare the construction projects and to select the most economically effective project implementation alternative in construction industry [30, 34]. LEVI 3.0 can evaluate the effects of the application of different methods of transformation on the numerical results and improve the quality of transformation as well as ensuring precise solving of technological and organizational problems (tasks). This application with usage of the appropriate transformation methods allows avoiding inaccuracies in assessing the alternatives of construction projects [6]. Therefore, all the information should be accumulated in the building databases for further construction and facility management. After completion of the analysis the following results were obtained (in both cases calculations done by competent executor and no influence of human factors were under consideration in made analysis):

Uncertainty management strategies presented in Table 2 involve an additional cognitive and behavioral process as opposed to basic risk management processes. In line with the strategies outlined, the project/safety manager could establish the required competencies in the project organization to manage particular uncertainty in refurbishment projects. Practicing these uncertainty management strategies in a project environment would help to keep the focus on project outcomes while performing in the midst of uncertainty. This potentially maintains a balance of safety and production. The ultimate utility of the uncertainty factor model and uncertainty management strategies lies in its ability to enable project professionals to identify the changing shape of risk due to any inherent project uncertainty at different project phases and subsequently develop the required capabilities within the project team to cope with unforeseen risks. Eventually, from a safety management perspective, this would potentially help to reduce the number of incidents and injury claims in a project, thus improving overall project safety performance.

\section{Summary and conclusions}

Uncertainty and risk management is still a problem area in the construction industry. In article, it was explained the appraisal procedures and solutions for decision making in construction under the conditions of risk and uncertainty. The following solutions were made to manage risk and uncertainty in construction during appraisal phase: The findings and patterns of this study, therefore, not only aid in enhancing the existing knowledge of project uncertainty in relation to construction refurbishment but also provides a comprehensive platform for the development of further studies in the field. Further, the factor model suggests that the proposed taxonomy has the ability to encourage uncertainty assessment and is a useful tool for industry practitioners in order to effectively manage project uncertainty 


\section{References}

[1] Hardie, M.; Miller, G.; Khan, S. Waste minimisation in office refurbishment projects: An Australian perspective. Open Waste Manag. J. 2011, 4, 21-27

[2] Ranasinghe, U.; Davis, P.; Pillay, M.; Jefferies, M. Uncertainty Induced Risks Influencing Safety Performance in Building Refurbishment projects: A Systematic Literature Review. In Proceedings of the CIB World Building Congress 2019, Hong Kong, China, 17-21 June 2019.

[3] Sezer, A.A.; Bosch-Sijtsema, P. Actor-to-actor tensions influencing waste management in building refurbishment projects: A service ecosystem perspective. Int. J. Constr. Manag. 2020, $1-10$.

[4] Uotila, U.; Saari, A.; Junnonen, J.-M. Uncertainty in the Early Phase of a Municipal Building Refurbishment Project-A Case Study in Finland. Buildings 2020, 10, 137.

[5] Ali, A.S.; Rahmat, I.; Noordin, N. Uncertainty in the design process of refurbishment projects. Built Environ. 2009, 6, 35-43.

[6] Saurin, T.A.; Rooke, J.; Koskela, L.; Kemmer, S. Guidelines for the management of complex socio-technical systems: An exploratory study of a refurbishment project. In Proceedings of the 21 st Annual Summit of the International Group for Lean Construction, Fortaleza, Brazil, 29 July-2 August 2013.

[7] Hughes, P.; Ferrett, E. Introduction to Health and Safety in Construction; Routledge: Abingdon, UK, 2012. 8. Sunil, S. Sustainable Refurbishment; John Wiley \& Sons, Ltd.: Chichester, UK, 2012. 